\title{
Programa Cuide-se Mais: impacto na prevenção e rastreamento do câncer no Paraná
}

\section{Take More Care Program: impact on cancer prevention and tracking in Paraná}

\author{
Thayse Fachin Cormanique ${ }^{1}$, Larissa Salla², Fabiana de Cássia Tozo ${ }^{3}$, \\ Ana Paula Vieira ${ }^{4}$, Lirane Elize Defante Ferreto ${ }^{5}$, Roberto Shigueyasu \\ Yamada $^{6}$, Franciele Ani Caovilla Follador ${ }^{7}$, Carolina Panis ${ }^{8}$
}

\begin{abstract}
Resumo
O aumento da incidência de câncer na população brasileira alerta para a necessidade de implantação de medidas de rastreamento baseadas na busca ativa de novos casos. Neste sentido, o presente trabalho avaliou o impacto de um programa itinerante de rastreamento dos cânceres de mama, colo de útero, próstata e pele realizado pelo Serviço Social da Indústria (Sesi) no período de 1 ano, em 22 municípios do estado do Paraná. O estudo é descritivo, com banco de dados secundários e abordagem quantitativa, referente ao programa Cuide-se Mais do Sesi Paraná. Através de uma unidade móvel, foram realizadas análises da superfície da pele, coleta de Papanicolau em mulheres de 25 a 69 anos, mamografia em mulheres com idade entre 40 e 69 anos e dosagem de Antígeno Prostático Específico (PSA) em homens de 45 a 69 anos de 22 municípios do Paraná. Foram atendidos 4679 trabalhadores paranaenses em 202 empresas, realizadas 4120 palestras de orientações e 1346 oficinas de prevenção. As lesões avaliadas em pele suspeitas para câncer corresponderam a 3,4\% do total de 3054 exames. Foram realizadas 873 mamografias, e encaminhados 11 casos de lesões suspeitas no exame de Papanicolau de um total de 2073 coletas. O PSA esteve alterado em 8 homens de um total de 1308 exames. A instalação da unidade móvel dentro da empresa quebra duas barreiras de impedimento dos trabalhadores realizarem seus exames preventivos: a sociocultural e a institucional. Desta forma, programas e ações de rastreamento do câncer podem contribuir para o diagnóstico precoce desta patologia, especialmente na população em idade economicamente produtiva.
\end{abstract}

Palavras-chave: Câncer. Rastreamento. Prevenção.

\footnotetext{
${ }^{1}$ Graduação em Medicina pela Universidade Estadual do Oeste do Paraná, Francisco Beltrão, Paraná, Brasil. Colaboradora no Laboratório de Biologia de Tumores do Centro de Ciências da Saúde da Universidade Estadual do Oeste do Paraná, Francisco Beltrão, Paraná, Brasil.

${ }^{2}$ Mestranda em Ciências Aplicadas à Saúde na Universidade Estadual do Oeste do Paraná, Francisco Beltrão, Paraná, Brasil.

${ }^{3}$ Especialização em Gestão de Projetos pela Faculdade de Ciências Sociais e Aplicadas do Paraná, Curitiba, Brasil. Coordenadora Técnica de Negócios do Serviço Social da Indústria do Paraná, Paraná, Brasil.

${ }^{4}$ Doutorado em Ciência de Alimentos pela Universidade Estadual de Campinas, Campinas, São Paulo, Brasil. Professora Associada do Centro de Ciências da Saúde da Universidade Estadual do Oeste do Paraná, Francisco Beltrão, Paraná, Brasil.

${ }^{5}$ Doutorado em Saúde Coletiva pela Universidade Estadual de Campinas, Campinas, São Paulo, Brasil. Professora Associada do Centro de Ciências da Saúde da Universidade Estadual do Oeste do Paraná, Francisco Beltrão, Paraná, Brasil.

${ }^{6}$ Mestre em Desenvolvimento Regional da Universidade Tecnológica Federal de Paraná, Pato Branco, Paraná, Brasil. Docente Assistente C do Centro de Ciências da Saúde da Universidade Estadual do Oeste do Paraná, Francisco Beltrão, Paraná, Brasil.

${ }^{7}$ Doutorado em Engenharia Agrícola - Recursos Hídricos e Saneamento Ambiental pela Universidade Estadual do Oeste do Paraná, Francisco Beltrão, Paraná, Brasil. Professora Associada do Centro de Ciências da Saúde da Universidade Estadual do Oeste do Paraná, Francisco Beltrão, Paraná, Brasil.

${ }^{8}$ Doutorado em Patologia Experimental pela Universidade Estadual de Londrina, Londrina, Paraná, Brasil. Professora Adjunta do Centro de Ciências da Saúde da Universidade Estadual do Oeste do Paraná, Francisco Beltrão, Paraná, Brasil. E-mail: carolpanis@hotmail.com
} 


\begin{abstract}
The increased incidence of cancer in the Brazilian population urges the need of implementing screening measures based on the active search for new cases. In this sense, the present work presents the results of the Take More Care disease-screening program. With a mobile unit, analyzes of the skin surface, Pap smear collection in women aged 25 to 69 years, mammography in women aged 40 to 69 years and specific prostatic antigen (SPA) in men aged 45 to 69 were performed in 22 municipalities of Paraná state. 4679 workers from Paraná were attended in 202 companies, 4120 orientation lectures and 1346 prevention workshops were given. Suspected skin lesions for skin cancer corresponded to $3.4 \%$ from 3054 skin assessment tests. 873 mammograms were performed, and 11 cases of suspected lesions were referred to the Pap smear, from 2073 collections. The SPA was altered in 8 men, in a total of 1308 tests performed. The installation of the mobile unit inside the company breaks two barriers preventing workers from carrying out their preventive exams: the sociocultural and the institutional. Thus, cancer screening programs and actions can contribute to the early diagnosis of this pathology, especially in the population of economically productive age.
\end{abstract}

Keywords: Cancer. Screening. Prevention.

\section{Introdução}

O câncer é uma patologia de origem multifatorial na qual o estilo de vida e as exposições ambientais são apontados como os principais fatores associados ao seu desenvolvimento. ${ }^{(1)} \mathrm{A}$ estimativa de novos casos de câncer para o Brasil em 2018-2019 segundo o Instituto Nacional de Câncer (INCA) é de aproximadamente $600 \mathrm{mil}$ casos, ${ }^{(2)}$ e até 2035, a Organização Mundial da Saúde (OMS) estima que o número de novos casos de câncer chegue a 24 milhões. ${ }^{(3)}$

Iniciativas de prevenção de doenças e promoção à saúde devem ser fortemente estimuladas, visando evitar a exposição a fatores de risco ambientais, bem como estimular mudanças no estilo de vida que possam minimizar o risco de patologias graves, como o câncer. A identificação precoce, o diagnóstico correto e o tratamento eficaz contribuem com o aumento da taxa de sobrevida do câncer e também auxiliam na redução do sofrimento do indivíduo e amplia as chances de cura. ${ }^{(4)}$

Neste sentido, programas e ações preventivas vêm sendo desenvolvidos no Brasil, como a Política Nacional de Prevenção e Controle do Câncer, o Programa Nacional de Controle do Câncer de Colo de Útero e o Programa Nacional de Controle do Tabagismo. ${ }^{(5)}$ Essas ações mudam o foco do tratamento para a prevenção, objetivando antecipar doenças futuras na população que se encontra saudável através do rastreamento da doença. ${ }^{(6,7)}$

Os testes utilizados para rastreamento de patologias como o câncer devem ser seguros e de boa relação custo-efetividade. Assim, o rastreamento da doença pode ser feito pela avaliação periódica e sistemática, como nos casos dos cânceres de colo de útero e mama, ou ainda através de avaliação clínica continuada e periódica, como nos casos da próstata e câncer de pele, e permite identificar lesões sugestivas de câncer na sua fase assintomática. ${ }^{(8)}$ Como exemplo, o uso da mamografia periódica como exame de rastreamento para o câncer de mama feminino possibilita o diagnóstico desta doença em estágios precoces e passíveis de tratamento, com elevadas chances de cura e redução da sua morbimortalidade. O rastreamento mamográfico pode reduzir a mortalidade por carcinoma mamário em até $25 \%$. $^{(9)}$

Resultados similares são observados em relação ao rastreamento do câncer de colo de útero, por meio da busca de mulheres com vida sexual ativa para realização do exame preventivo de Papanicolau. De acordo com o Instituto Nacional de Câncer, tanto a incidência como a mortalidade por câncer de útero podem ser reduzidas com o rastreamento, ${ }^{(10)}$ uma vez que tal procedimento permite a identificação das lesões precursoras do mesmo. 
Embora existam diversos programas voltados à detecção precoce do câncer, seu alcance ainda é limitado pela dimensão geográfica do Brasil, o que aponta para a necessidade de ações que alcancem munícipios no interior dos estados do País através do mecanismo de busca ativa de casos visando a detecção precoce desta patologia. O câncer tem altas chances de cura e evolução com bom prognóstico, quando detectado nos estágios iniciais. ${ }^{(11)}$ Neste contexto, o presente trabalho avaliou o impacto de um programa itinerante de rastreamento dos cânceres de mama, colo de útero, próstata e pele realizado pelo Serviço Social da Indústria (Sesi) no período de 1 ano, em 22 municípios do estado do Paraná.

\section{Metodologia}

Realizou-se estudo descritivo de banco de dados secundários, com abordagem quantitativa, referente ao programa Cuide-se Mais do Sesi Paraná. O programa Cuide-se Mais foi promovido pelo Sesi do Paraná na forma de prestação de serviços, com o intuito de promover a qualidade de vida para os trabalhadores do estado do Paraná através de ações de educação continuada, prevenção e diagnóstico no ano de 2014. O programa foi dividido em oito eixos, sendo a Prevenção do Câncer uma das áreas temáticas. Por meio de uma unidade móvel, foram realizadas consultas médicas para avaliação do estado geral de saúde e a coleta de material para exames, através das seguintes condutas:

- Análise da superfície da pele para identificação de lesões sugestivas de câncer de pele;

- Coleta de exames preventivos de câncer uterino para realização de Papanicolau em mulheres de 25 a 69 anos;

- Realização de exames de mamografia em mulheres com idade entre 40 e 69 anos;

- Coleta de sangue periférico para dosagem dos níveis de Antígeno Prostático Específico (PSA), para rastreamento do câncer de próstata em homens de 45 a 69 anos.
O material coletado foi encaminhado para os laboratórios e hospitais credenciados do programa, sendo eles: Hospital de Barretos (Barretos, São Paulo), Centro de Hematologia e Hemoterapia do Paraná (Hemepar, Curitiba, Paraná), Hospital Pequeno Príncipe (Curitiba, Paraná), Universidade Estadual do Oeste do Paraná, campus Francisco Beltrão (Unioeste, Francisco Beltrão, Paraná), Hospital João de Freitas (Arapongas, Paraná), Hospital do Câncer de Cascavel (Uopeccan, Cascavel, Paraná), Centro de Oncologia de Cascavel (CEONC, Cascavel, Paraná) e Hospital do Câncer de Londrina (Londrina, Paraná). A conduta adotada pelos organizadores do rastreamento do câncer ao identificar um caso positivo foi de encaminhar para o tratamento, e aos demais foram ofertadas palestras e oficinas sobre o tema. Como se trata de uma pesquisa de banco de dados secundários de consulta pública conforme a Resolução CNS 466/12, exclui-se a necessidade de aprovação de um comitê de ética em pesquisa.

\section{Resultados}

O programa Cuide-se Mais foi realizado no período de janeiro a dezembro de 2014, e visitou 22 municípios paranaenses (área total de aproximadamente $40.000 \mathrm{~km}$ [Figura 1]). O programa atendeu trabalhadores de empresas que não possuem planos privados de saúde ou têm dificuldade de acesso aos procedimentos listados. Os setores atendidos englobam trabalhadores da área têxtil, madeira e mobiliário, construção civil, eletroeletrônicos, alimentício, comércio, gráfico, educação, mineral, químico, borracha e materiais plásticos.

Os municípios atendidos pelo programa Cuide-se Mais foram: Marechal Cândido Rondon, Arapongas, Cascavel, Foz do Iguaçu, Capanema, Planalto, Realeza, Ampére, Dois Vizinhos, Enéas Marques, Santo Antonio do Sudoeste, Francisco Beltrão, Marmeleiro, Pato Branco, Barro Preto, Piraí do Sul, Ponta Grossa, Araucária, Campo Largo, São José dos Pinhais, Curitiba e Palmas, conforme pode se observar na Figura 1. 
Figura 1 - Municípios paranaenses atendidos pelo projeto Cuide-se Mais, no período de janeiro a dezembro de 2014.

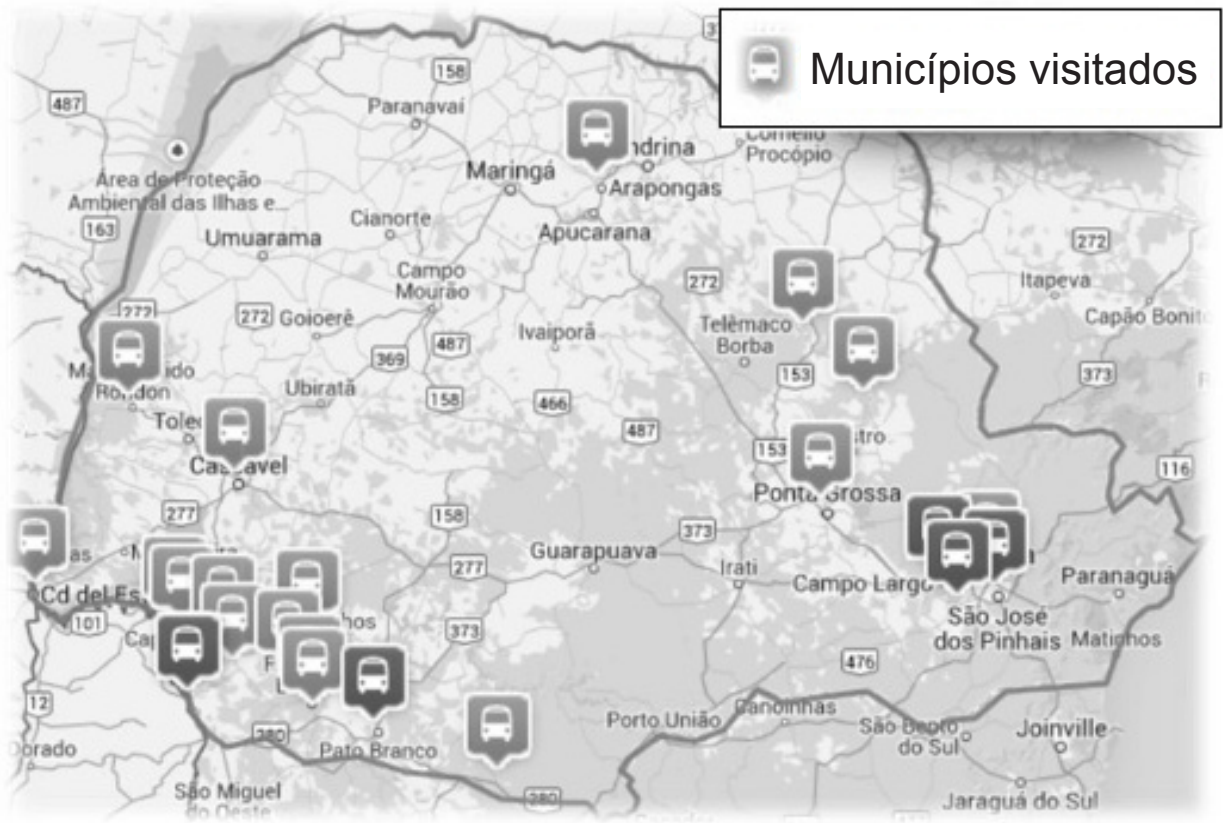

Fonte: Dados da pesquisa

Conforme demonstrado na Tabela 1, a unidade móvel teve o alcance de atendimento em 202 empresas, atingindo um número total de 4679 trabalhadores paranaenses (sendo 44\% homens e $56 \%$ mulheres), o que correspondeu a
$34,36 \%$ do total de trabalhadores destas empresas. Neste público, foram realizadas 4120 palestras de orientações e 1346 oficinas de prevenção, abordando temáticas relacionadas à qualidade de vida e prevenção de câncer.

Tabela 1 - Público atendido pelo projeto Cuide-se Mais do Sesi, no estado do Paraná, no período de janeiro a dezembro de 2014.

\begin{tabular}{lc}
\hline & Total \\
\hline Empresas envolvidas no projeto & 202 \\
Palestras de orientação & 4120 \\
Oficinas de prevenção & 1346 \\
Trabalhadores atendidos & 4679 \\
- Homens & $44 \%$ \\
- Mulheres & $56 \%$ \\
\hline
\end{tabular}

Fonte: Autores

Conforme se observa na Tabela 2, foram realizados 3054 exames de avaliação de pele. Destes, 104 indivíduos foram encaminhados para realização de exames complementares; desta forma, $3,4 \%$ dos trabalhadores atendidos apresentavam lesões de pele suspeitas para câncer de pele, dos quais um caso foi confirmado. Também foram realizadas 873 mamografias, o que indica que mais de $50 \%$ da população feminina atendida realizou o exame de rastreamento do câncer de mama. 
Tabela 2 - Perfil dos exames realizados pelo projeto Cuide-se Mais no estado do Paraná, no período de janeiro a dezembro de 2014.

\begin{tabular}{lc}
\hline & Total \\
\hline Avaliações de pele & 3054 \\
Número de exames realizados & 104 \\
Encaminhamento para exames complementares & 1 \\
Diagnóstico de câncer de pele & \\
Mamografia & 873 \\
Número de exames realizados & 11 \\
Encaminhamento para exames complementares & 1 \\
Diagnóstico de câncer de mama & \\
Exame de Papanicolau & 2073 \\
Número de exames realizados & 11 \\
Encaminhamento para exames complementares & 4 \\
Diagnóstico de câncer de útero & \\
Exame de próstata - Antígeno Prostático Específico (PSA) & 1308 \\
Número de exames realizados & 8 \\
Encaminhamento para exames complementares & 1 \\
Diagnóstico de câncer de próstata & $\mathbf{7 2 7 1}$ \\
Total de exames realizados & \\
\hline
\end{tabular}

Fonte: Autores

Em relação ao exame de Papanicolau, foram realizadas 2073 coletas, com 11 encaminhamentos de lesões suspeitas para procedimentos confirmatórios e quatro diagnósticos confirmados de câncer de útero. $\mathrm{O}$ exame para rastreamento de câncer de próstata foi realizado em 1308 homens (18\% do total de trabalhadores atendidos), resultando em oito encaminhamentos para exames confirmatórios e um diagnóstico confirmado de câncer prostático. Desta forma, o total de exames realizados para rastreamento dos cânceres em questão foi de 7271 .

\section{Discussão}

A conscientização dos trabalhadores sobre a importância da prevenção de doenças crônicodegenerativas vem sendo o principal objetivo dos programas de rastreamento no Brasil. Neste sentido, a proposta do programa Cuide-se Mais visa avaliar por meio de exames de rotina os trabalhadores que apresentam potencial risco de desenvolvimento de diversas patologias, incluindo o câncer. Sabe-se que o diagnóstico precoce do câncer permite que a doença seja rapidamente tratada, com chance de cura superior a $90 \%$. Além disso, estima-se que ações de prevenção e detecção precoce do câncer possam prevenir cerca de um terço das mortes por esta patologia. ${ }^{(12)}$ Assim, programas de prevenção que atinjam a população em idade produtiva e que não têm fácil acesso aos serviços de saúde ainda são escassos no Brasil, sendo ainda mais relevantes quando ofertados próximo ou no ambiente de trabalho. Ações de saúde no ambiente de trabalho têm melhor adesão do trabalhador já que são realizadas em espaços organizacionais que o indivíduo passa grande parte do seu dia, evita os deslocamentos e horas fora da atividade laboral, além de proporcionar ao trabalhador sensações agradáveis, seguras e estimulantes de valorização do seu bem-estar por parte da sociedade, ${ }^{(13)}$ contribuem para detecção, 
controle e enfraquecimento de fatores de risco relacionados a doenças nessa população. ${ }^{(14)}$

Neste cenário, o programa Cuide-se Mais desponta como uma das principais iniciativas de rastreamento precoce de doenças crônicas no Paraná. Foram escolhidos para rastreamento os quatro cânceres de maior incidência na população brasileira e no estado do Paraná: próstata, mama, útero e pele. ${ }^{(2)}$

Os cânceres de próstata e de mama estão entre as neoplasias que mais matam no Brasil. ${ }^{(2,15)}$ $\mathrm{O}$ câncer de próstata é o segundo tipo mais frequente na população masculina, acometendo principalmente homens acima dos 65 anos, ${ }^{(16)}$ e seu rastreamento se dá através do teste de dosagem do antígeno prostático específico no sangue, realizado em homens a partir dos 40 anos. ${ }^{(17)}$ Dentro do programa foram rastreados 1308 homens com idade entre 45 e 69 anos, dos quais oito apresentaram alterações e foram encaminhados para realização de exames adicionais, onde um caso foi confirmado como câncer de próstata e encaminhado para tratamento.

Ainda não existe um consenso sobre os benefícios da realização de um programa de rastreamento de câncer de próstata na população, e por conta disso não existem políticas públicas de incentivo ao rastreamento em massa do câncer de próstata por dosagem de PSA na população. ${ }^{(18)}$ A dosagem de PSA ainda não é consenso para detecção do câncer de próstata devido ao seu amplo nível plasmático, que varia de 3 a $10 \mathrm{ng} / \mathrm{mL}$ no sangue de homens saudáveis, ${ }^{(19)}$ o que dificulta o estabelecimento de um ponto de corte adequado que permita a identificação da doença clínica. De acordo com o consenso do Programa Nacional de Controle do Câncer de Próstata, considerando-se o ponto de corte de $4 \mathrm{ng} / \mathrm{mL}, 72 \%$ dos homens apresentariam níveis aumentados de PSA e estariam sujeitos à realização de biópsia. ${ }^{(18)}$ Por outro lado, podem existir tumores de próstata com valores de PSA abaixo de $4 \mathrm{ng} / \mathrm{mL}$. A indicação formal de biópsia ocorre quando o PSA se encontra acima de $10 \mathrm{ng} / \mathrm{mL}$. Apesar disso, ações preventivas são encorajadas visando à detecção precoce, aumento da chance de cura e preservação da qualidade de vida masculina. Ainda existem crenças e barreiras conceituais sobre o tratamento de câncer de próstata. ${ }^{(20)}$ Com relação às crenças sobre a doença: possibilidade de vida normal embora doente, que pode ser assintomático, o tratamento é pior que a doença e o exame do toque retal afeta a masculinidade e, se estiver bem, não é necessário fazê-lo. Quanto às barreiras: o médico nunca solicitou, não acham importante e têm medo de fazer o exame. $\mathrm{O}$ comportamento e as práticas em relação ao câncer de próstata em adultos apresentam comportamento não preventivo, sendo que o primeiro contribui na indicação medo da presença da doença e o segundo avalia a próstata quanto ao seu tamanho, consistência e se há nódulos, ou seja, a associação dos dois aumenta a sensibilidade do diagnóstico das doenças prostáticas. ${ }^{(21)}$ Dor, a vergonha e a falta de coragem para realizar o exame constituem barreiras a esses indivíduos em procurar os serviços de assistência à saúde. ${ }^{(22)}$

Em relação ao câncer de mama, das mamografias realizadas, 11 foram encaminhadas para exames complementares e uma foi diagnosticada como câncer de mama. $O$ câncer de mama é a neoplasia maligna que mais mata mulheres no mundo, principalmente devido ao diagnóstico da doença em estágios avançados. Estima-se que a realização de mamografia periódica em mulheres acima dos 50 anos possa evitar cerca de $30 \%$ das mortes associadas ao câncer de mama. ${ }^{(23)} \mathrm{O}$ documento de consenso sobre o Controle de Câncer de Mama no Brasil (24) recomenda o rastreamento do câncer de mama em mulheres entre 50 e 69 anos por meio da realização de mamografia anual em associação ao exame clínico das mamas. Embora existam programas públicos de rastreamento da doença, o acesso às mamografias pelas mulheres ainda é deficiente. Dados da Sociedade Brasileira de Mastologia e do Instituto Nacional de Câncer indicam que o programa de rastreamento do câncer de mama do Ministério da Saúde (MS), apesar dos esforços contínuos, tem falhas de alcance importantes. ${ }^{(23,24)}$ 
Diante disso, foi anunciada a possibilidade da criação de unidades móveis de mamografia pelo governo, o que enfatiza a importância de iniciativas de busca ativa como o programa Cuide-se Mais. Este dado foi corroborado pelo nosso estudo, pois apesar de a Unidade Móvel de Mamografia estar presente nas empresas oferecendo o serviço gratuitamente e com consulta médica, apenas $52 \%$ do total de mulheres das empresas fizeram o exame.

Em relação aos cânceres de mama, ainda existem barreiras conceituadas que dificultam o acesso da mulher a serviços de saúde durante a condução do caso de câncer de mama, como a própria mulher, familiares, amigos, profissionais de saúde, instituição ou serviço. ${ }^{(25)}$ No rastreamento do câncer de colo uterino, quatro casos foram confirmados como positivos para câncer. A neoplasia uterina é uma das poucas patologias que se pode prevenir em oncologia, através da realização do exame preventivo anual de Papanicolau. O Programa Nacional de Rastreamento do Câncer de Colo de Útero estima que cerca de $80 \%$ da população feminina em idade sexualmente ativa esteja coberta pela realização do exame preventivo, entretanto nossos dados apontam para uma possível falha do programa nas regiões atendidas, seja por falta de adesão das mulheres ou de cobertura pela rede assistencial. ${ }^{(2)}$

A pesquisa ${ }^{(1)}$ revelou que as barreiras para não realização do exame Papanicolau foram: conhecimento insuficiente, sentimentos negativos, falta de atitude, aspectos vinculados aos serviços de saúde e inserção da mulher no mercado de trabalho. Ainda verificaram que ficou evidente que a interação e interdependência dessas barreiras vão interferir sinergicamente no processo de adesão à realização do exame Papanicolau. Portanto, observa-se a necessidade de intervenções mais humanizadas, equitativas e focadas em eliminar as barreiras e iniquidades no acesso e utilização dos serviços preventivos, respeitando as realidades intrínsecas de cada mulher. Os sentimentos de vergonha e questões culturais, além de falta de recursos materiais, conhecimento da rotina do serviço e informações e esclarecimentos durante a consulta ginecológica são motivos para que as mulheres não façam o exame com a periodicidade desejada. $^{(1,2)}$

É importante salientar a importância das oficinas de prevenção e palestras de orientação dentro das empresas atendidas, pois todos os cânceres abordados neste estudo são patologias com possibilidade de prevenção e cura nas fases iniciais da doença. A associação entre rastreamento, diagnóstico precoce e educação continuada pode ajudar de forma significante a reduzir a incidência e a mortalidade por câncer no Brasil.

\section{Conclusão}

A utilização do projeto "Cuide-se Mais" promovido pelo Sesi do Paraná consiste em uma estratégia complementar dentro da Política Nacional de Enfrentamento das Doenças Crônicas Degenerativas no Brasil, pois atua nas duas barreiras que dificultam os trabalhadores a realizarem seus exames preventivos de doenças: a quebra da barreira sociocultural, através de palestras de orientações e oficinas de prevenção, para conscientização da importância da promoção da saúde e prevenção de doenças; e a quebra da barreira institucional com a instalação para estas atividades dentro da própria empresa com a unidade móvel.

\section{Conflitos de interesse}

Os autores declaram que não há conflitos de interesse.

\section{Referências}

1 Aguilar RP, Soares DA. Barreiras à realização do exame Papanicolau: perspectivas de usuárias e profissionais da Estratégia de Saúde da Família da cidade de Vitória da Conquista-BA. Physis Rev de Saúde Coletiva. 2015 Fev; 359:379-425. doi.org/10.1590/S0103-73312015000200003.

2 Instituto Nacional De Câncer. Estimativa 20182019: Incidência de Câncer no Brasil. Rio de Janeiro: INCA; 2014. 
3 Cotter T, Perez D, Dessaix A, Baker D, Murphy M, Crawford J, et al. Cancer and Lifestyle Factors. Sydney: Cancer Institute; 2007.

4 Nascimento RG, Araújo A. Falta de periodicidade na realização do exame citopatológico do colo uterino: motivações das mulheres. Rev Min Enferm. 2014 Jul-Set;18(3):557-72. doi. org/10.5935/1415-2762.20140041.

5 Radis. Casos de câncer aumentam no mundo. Radis 138 [Internet]. 2014 [citado 2019 set 21] Disponível em: www.ensp.fiocruz.br/radis

6 Ferreira ML, Oliveira C. Conhecimento e significado para funcionárias de indústrias têxteis sobre prevenção do câncer do colouterino e detecção precoce do câncer da mama. Rev Bras Cancerol. 2006;52(1): 5-15.

7 Instituto Nacional De Câncer. Política Nacional de Prevenção e Controle do Câncer. Rio de Janeiro: INCA; 2015.

8 Andrade ALSS, Martelli CMT, Pinheiro ED, Santana CLB, Borges FP, Zicker F. Rastreamento sorológico para doenças infecciosas em banco de sangue como indicador de morbidade populacional. Rev Saúde Publ.1989;23(1):20-5. doi: 10.1590/S003489101989000100004.

9 Lima ALP, Rolim NCOP, Gama MEA, Pestana AL, Silva EL, Cunha CLF. Rastreamento oportunístico do câncer de mama entre mulheres jovens no Estado do Maranhão, Brasil. Cad. Saúde Públ. 2011; 27(7): 1433-9.

10 World Health Organization. Cancer Control. Knowledge into action. WHO guide for effective programs. Early Detection Module. Switzerland: WHO; 2007.

11 Gotzsche PC, Nielsen M. Screening for breast cancer with mammography. Cochrane Database Syst Veer. 2006;6:CD001877.

12 Gotzsche PC, Nielsen M. Screening for breast cancer with mammography. Cochrane Database Syst Ver. 2013; 6:CD001877. doi: 10.1002/14651858.CD001877.pub5.

13 Cavalcante CAA, Nóbrega JAB, Enders BC, Medeiros SM. Promoção da saúde e trabalho: um ensaio analítico. Rev Eletronica de Enfermagem. 2008; 10(1):241-8. doi. org/10.5216/ree.v10i1.8017.

14 Pinafo E, Nunes EFPA, Gonzalez AD. A educação em saúde na relação usuáriotrabalhador no cotidiano de equipes de saúde da família. Ciênc. Saúde Coletiva [Internet]. 2017 [citado 2020 maio 28]; 1825:1832-7. Disponível em: http://www.scielo.br/scielo. php?script=sci_arttext\&pid=S1413-

15 Instituto Nacional De Câncer. Controle do Câncer de Colo de útero. Rio de Janeiro: INCA; 2019.

16 Instituto Nacional De Câncer. ABC do câncer: abordagens básicas para o controle do câncer. Rio de Janeiro: INCA; 2011.

17 Vieira SC, De Brito LXE, Soares LFM, Teixeira JM, Lustosa AML, Barbosa CNB et al. Oncologia Básica, Teresina, PI: Fundação Quixote; 2012.

18 Cecilio APB, Takakura ET, Jumes JJ, Santos JW, Herrera AC, Victorino VJ, et al. Breast cancer in Brazil: epidemiology and treatment challenges. Breast Cancer Targets Ther. 2015;7:43-9.

19 Gomes R, Rebello LEFS, Araújo FC, Nascimento EFA. Prevenção do câncer de próstata: uma revisão de literatura. Ciênc. \& Saúde Col. 2008;13(1):235-46. doi. org/10.1590/S1413-81232008000100027.

20 Paiva EP, Motta MCS, Griep RH. Barreiras em relação aos exames de rastreamento do câncer de mama: percepção de mulheres. Rev Esc Enferm USP. 2014; 48(3):394-400.

21 Belinelo RGS, Almeida SM, Oliveira PP, Onofre PSC, Viegas SMF, Rodrigues AB. Exames de rastreamento para câncer de próstata: vivência de homens. Esc Anna Nery Rev de Enfermagem. 2014. 18(4):697-704. doi.org/10.5935/1414-8145.20140099.

22 Menezes R, Menezes M, Teston EF, Matumoto S, Faller JW. Conhecimento, comportamento e práticas em saúde do homem em relação ao câncer de próstata. Rev Pesqui. 2019 outdez;11(5):1173-9 
23 Martins ACP, Monti PR, Rodrigues PRM, Ponte JRT, Fonseca AG. Câncer de Próstata: prevenção e rastreamento. Projeto Diretrizes da Sociedade Brasileira de Urologia [Internet]. 2006 [citado 2018 jan 21]. Disponível em: www.projetodiretrizes.org.br/5_volume/10CancerPrev.pdf

24 Instituto Nacional De Câncer. Programa Nacional De Controle Do Câncer Da Próstata: Documento De Consenso. Rio de Janeiro: INCA; 2020.

25 Gonçalves LLC, Travassos GL, Almeida AM, Guimarães AMDN, Gois CFL. Barreiras na atenção em saúde ao câncer de mama: percepção de mulheres. Rev Esc Enferm USP [Internet]. 2014 [citado 2020 maio 28]; 48(3):394-400. Disponível em www.ee.usp.br/ reeusp/ 
